ACTA UNIVERSITATIS LODZIENSIS

Folia Litteraria Romanica 15, 2020

https://doi.org/10.18778/1505-9065.15.15

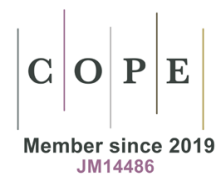

Tomasz Kaczmarek

Université de Lodz

iD ORCID ID : 0000-0001-6138-5280

tomasz.kaczmarek@uni.lodz.pl

\title{
Le théâtre français de contestation sociale autour de 1900 : l'exemple de Dégénérés ! de Michel Provins
}

\begin{abstract}
RÉSUMÉ
Dégénérés! de Michel Provins est une œuvre particulière dans le répertoire du théâtre français de contestation sociale. Inspiré par les traités médicaux sur les cas de " dégénérescence », le dramaturge décide d'y brosser les portraits de bourgeois égoïstes et pervers qui, au lieu de remplir leurs obligations envers la société, s'enlisent dans une vie de débauche. L'écrivain exprime un mépris sincère à l'égard des élites moralement abaissées qui contribuent à la dégradation de toute la nation. Tout d'abord, il fustige les travers des politiciens qui font carrière aux dépens des plus démunis, puis, il dénigre les hommes d'affaires et banquiers qui s'enrichissent outrageusement par la spéculation, et enfin, il s'en prend aux artistes et littérateurs qui propagent des vices dévastateurs pour la santé publique. En dépit de son langage virulent, Provins ne pense pas à se rallier aux anarchistes, il veut bouleverser les consciences afin de régénérer l'humanité.
\end{abstract}

MOTS-CLÉS - Michel Provins, théâtre de contestation sociale, mépris, haine, dénigrement, dégénérescence

\footnotetext{
"The French Theatre of Social Protest around 1900: the Example of Degenerates! by Michel Provins"
}

\begin{abstract}
SUMMARY
Degenerates! by Michel Provins is a particular work in the repertoire of the French theatre of social protest. Inspired by the medical treatises on cases of "degeneration", the playwright decides to paint the portraits of selfish and perverse bourgeois who, instead of fulfilling their obligations to society, get bogged down in a life of debauchery. The writer expresses his sincere contempt for morally bankrupt elites who contribute to the degradation of the whole nation. First of all, he castigates politicians who make a career out of the poor, then he denigrates businessmen and bankers who enrich themselves outrageously by speculation, and finally, he attacks the artists and writers who propagate devastating vices for public health. Despite his virulent language, Provins does not think of allying with the anarchists, he wants to stir the consciences in order to regenerate humanity.
\end{abstract}

KEYWORDS - Michel Provins, theatre of social protest, contempt, hatred, denigration, degeneration 
Le simple exposé de la théorie des dégénérescences suffit déjà pour faire entrevoir l'idée qu'il est permis de se faire de l'aliénation mentale. Il est incontestable : $1^{\circ}$ que lorsque cette maladie est le résultat de phénomènes pathologiques transmis par hérédité, elle constitue un véritable état dégénératif ; $2^{\circ}$ que lorsqu'elle est un fait primitif on a toute raison de craindre que, dans les générations subséquentes, elle ne se caractérise aussi par la dégradation de la race, et finalement par son extinction ${ }^{1}$.

Bénédict Augustin Morel

Le théâtre de contestation sociale ${ }^{2}$, qui jouit d'une renommée incontestable auprès du public de plus en plus conscient des inégalités du régime capitaliste au tournant du $\mathrm{XX}^{\mathrm{e}}$ siècle, fustige sans pitié les vices des bourgeois, perçus comme de féroces exploiteurs. Qu'il s'agisse de Jean Grave, de Georges Darien ou de Lucien Descaves, écrivains considérés comme anarchistes, ils accusent la Troisième République de toutes les injustices sociales. Il y en a qui, tel Octave Mirbeau dans Les Farces et moralités (1904), recourent à une satire caustique pour dénoncer la cruauté et la bêtise de la classe sociale privilégiée. On pourrait évoquer aussi à ce propos Charles Malato dont Barbapoux (1900), rappelant par son vocabulaire scatologique Ubu roi (1896) d'Alfred Jarry, arrache les masques aux protecteurs de l'ordre réactionnaire, tout en comparant le monde politique à des latrines. Les auteurs attachés à l'esthétique naturaliste n'hésitent pas non plus à vilipender les mœurs de l'establishment de l'époque. Pour se rendre compte des sujets abordés par maints écrivains, il suffit d'en citer quelques-uns : Henry Becque qui critique les gens de finance (Les Polichinelles ${ }^{3}$ ), Eugène Brieux qui condamne la charité des bourgeois comme remède aux maux sociaux (Les Bienfaiteurs, 1896), ainsi que Georges Ancey qui s'en prend au clergé (Ces Messieurs, 1901). Parmi les nombreux textes dénigrant ouvertement les oppresseurs, une pièce au titre aussi éloquent que provocateur Dégénérés ! ${ }^{4}$ (1897) mérite une attention particulière. S'inspirant des travaux des chercheurs de son époque tels que Lombroso, Nordau, Morel ou Legrain, Michel Provins (nom de plume d'Anne Gabriel François Camille Lagros de Langeron, 1861-1928) brosse dans son drame des portraits d'individus

B.A. Morel, Traité des maladies mentales, Paris, Librairie Victor Masson, 1860, p. 4.

2 Le Théâtre de contestation sociale autour de 1900, textes réunis et présentés par J. Ebstein, J. Hughes, $\mathrm{Ph}$. Ivernel. M. Surel-Tupin, PUBLISUD, 1991 ; Au temps de l'anarchie, un théâtre de combat : 1880-1914, choix et édition des textes par J. Ebstein, Ph. Ivernel, M. Surel-Tupin et S. Thomas, Séguier / Archimbaud, 2001, 3 vol. Pour en savoir plus : W. Asholt, Gesellschaftkritisches Theater im Frankreich der Belle Époque (1887-1914), Studia Romanica 59, Heidelberg, Carl Winter Universitätsverlag, 1984. À propos du théâtre français au tournant du $\mathrm{XX}^{\mathrm{e}}$ siècle consulter : M. Autrand, Le Théâtre en France de 1870 à 1914, Paris, Honoré Champion, 2006.

3 C'est Henri de Noussane qui va écrire Les Polichinelles (1910), pièce tirée du manuscrit inachevé d'Henry Becque.

4 La pièce se compose d'un texte principal en trois actes, suivi de quelques « dialogues », comme l'auteur les appelle, dont le dénominateur commun est la critique acerbe de la société bourgeoise de la fin du XIX $\mathrm{X}^{\mathrm{e}}$ siècle : Un déjeuner de chasse, Genèse d'un socialiste, Un mouvement administratif, Recommandations! Un firt, La veillée des noces, l'Anniversaire, Un salon parisien en 1897. 
pervers et hideux qui, malgré leur défaillance mentale et morale, s'octroient le droit de représenter l'élite de la société. L'étude de ces « spécimens humains » est tout de même dépourvue de neutralité scientifique car le dramaturge ne cache pas ses sentiments critiques à l'égard de ses personnages. Dans les descriptions minutieuses, qui ne sont pas sans rappeler l'exactitude des traités médicaux, l'ironie se mêle constamment tantôt à la colère, tantôt à la haine. Sans aucun doute l'écrivain exprime ainsi son mépris sincère envers ces pantins incapables de remplir dignement leur fonction dans la vie sociale. Il est intéressant à ce propos de se pencher sur les procédés auxquels l'auteur a recours pour rendre son jugement sévère sur la conduite moralement condamnable et indigne d'estime de ses « dégénérés ».

Issu d'une famille aisée, Michel Provins est fortement attaché à sa caste dont il semble un représentant exemplaire. Licencié en droit, il grimpe les échelons de la carrière sociale. Secrétaire de Waldeck-Rousseau, administrateur de plusieurs sociétés financières, entre autres, de la Banque Parisienne, directeur de l'Illustration puis fondateur de l'Illustration Économique et Financière, nommé chevalier de la Légion d'honneur, la vie de Provins illustre son ascension dans la société bourgeoise. Il a été amené à la littérature par le goût qu'il a toujours professé pour les lettres. Au demeurant, son milieu l'y disposait, car son père, préfet sous l'Empire, était aussi écrivain. Aujourd'hui inconnu, Provins jouissait de son temps de la renommée d'un observateur profond et satiriste impitoyable de la société française. On reconnaît aussitôt dans ses œuvres une ironie et une verve polémique aussi malicieuse que mordante. Son premier volume, Lettres d'homme (1892), qui remporte un succès incontestable, le confirme en tant qu'écrivain. Suivent, entre autres, La Femme d'aujourd'hui (1895) ou Les Lendemains d'aujourd'hui (1899). Dans le premier texte, l'auteur décrit la compagne de l'homme comme un être foncièrement déraisonnable, ce qui vient du simple fait que « la femme a été faite trop vite $»^{5}$. Au demeurant, le devoir de la femme normale est, aux yeux de l'écrivain, d'obtempérer à son rôle de mère. Ce motif réapparaît dans Le Fond secret (1905) où Provins se moque du mariage «bien parisien » ou dans Celles qu'on brûle, celles qu'on envoie (1911), où il dénonce les hypocrisies du cœur, tout en se concentrant sur les stratégies de la femme qui " est instinctivement comédienne $»^{6}$, et, enfin, dans un des derniers textes : Tendresses futiles (1923), où il s'adonne à l'étude de la femme en mettant à nu les tendresses du cœur féminin. Ses romans, jugés dialogués, trahissent son faible pour les pièces de théâtre. Il fait ses premières armes en tant que dramaturge en écrivant Dégénérés! Après ce succès, il se dédie à la composition de plusieurs drames dans lesquels, comme dans sa prose, il tente de révéler les déviations sociales et les mœurs corrompues. Il est indigné par l'immoralité des classes dirigeantes et des artistes, stigmatise le mariage d'intérêt bourgeois, s'insurge contre l'éducation matérialiste. Sans prétendre à l'exhaustivité,

5 M. Provins, La Femme d'aujourd 'hui, Paris, Victor-Havard, 1895, p. 1.

6 M. Provins, «L'école des flirts », L'Art et la scène, n 12, 1897, p. 3. 
citons, parmi tant d'autres, quelques titres des œuvres abordant ces questions qui ont fait le tour de l'Europe et de l'Amérique : L'École des flirts, 1899; Le Vertige, 1901 ; Les Arrivistes, 1903 ; Le Désir, 1905.

Face à la caricature de la bourgeoisie, il serait, pourtant, erroné de considérer Provins comme un écrivain de la génération contestataire. Il est plutôt un auteur de comédies critiques dans la tradition de Molière. Son thème fondamental est l'époque de la Troisième République. En la décrivant, il désire pourfendre des abus, dénoncer les mœurs, dénigrer l'art et la littérature qui, au lieu d'instruire, détruisent moralement les esprits. Il ne cesse de consacrer son théâtre à l'édification de la société bourgeoise tout en croyant à sa régénération. Dès lors, il n'envisage pas le renversement du régime social, car il espère, au travers de son œuvre, signaler les travers, réformer les injustices et corriger les vices. D'après Édouard Beaudu, Provins

est un joyeux moraliste. Il se moque agréablement de ses contemporains parce qu'il pense sans doute que la meilleure façon de ne pas paraître égoïste est encore celle-là. Il se permet de donner quelques leçons à ceux qui trouvent leurs prototypes parmi les personnages de son Guignol. Au fond, M. Michel Provins doit aimer passionnément la vertu pour se plaire tant à peindre les vices ${ }^{7}$.

Quand Jean Grave (1854-1939), militant anarchiste ${ }^{8}$, choisit de publier des extraits ${ }^{9}$ de la pièce ${ }^{10}$ de Provins, il entend toujours « fourrer des idées dans la tête des individus $\rangle^{11}$ pour les sensibiliser aux excès du système capitaliste monstrueux. De fait, en lisant les dialogues dans lesquels l'écrivain blâme sévèrement les bourgeois, il serait légitime d'y voir la verve subversive d'un antiautoritaire intraitable. Néanmoins, l'auteur du Fétichisme de la loi ne sélectionne à bon escient que les fragments qui pourraient contribuer au combat contre les possesseurs tout en passant sous silence les convictions profondément conservatrices de l'écrivain. Qui plus est, Grave n'aurait pas pu donner au public l'avertissement qui précède le drame de Provins où celui-ci s'explique longuement sur ses vraies intentions créatrices qui sont en nette opposition avec les postulats des contestataires. Or, si le dramaturge assène des coups durs à la bourgeoisie oisive, ce n'est pas qu'il rêve tout simplement de la supprimer, mais il désire ébranler les esprits de son époque

\footnotetext{
7 É. Beaudu, « Nouvelle Fantaisies », La Chronique des livres, 10-15 juillet 1904, p. 174.

8 Cf. L. Patsouras, Anarchism of Jean Grave, Black Rose Books, 2001 ; M. Delfau, Quarante ans de propagande anarchiste, Flammarion, 1973 ; J. Maitron, Le Mouvement anarchiste en France, Gallimard, 1992.

9 Grave proposait à ses lecteurs des extraits de certaines œuvres littéraires ou pièces de théâtre d'auteurs de diverses convictions politiques, in extenso si elles étaient courtes, dans le supplément littéraire de La Révolte et puis des Temps nouveaux.

${ }^{10}$ Grave publie les extraits tirés du drame principal ainsi que l'intégralité des deux « dialogues »: Un déjeuner de chasse et Un mouvement administratif.

${ }^{11}$ Les Temps nouveaux, 12-16 décembre 1896.
} 
afin de préserver l'ordre public affaibli par la dégradation « anthropologique aux variations maladives de l'espèce humaine $\rangle^{12}$.

Dans la deuxième moitié du XIX ${ }^{\mathrm{e}}$ siècle naissent diverses théories ${ }^{13}$ sur le « mal » qui commence à saccager sournoisement l'Occident. Bénédict Augustin Morel (1809-1873) ne tarde pas à ce propos à lancer la formule catastrophiste de dégénérescence ${ }^{14}$ de la race qui s'étend d'une génération à l'autre. Dès lors, le mot fait carrière. On met en garde dès 1855 sur le fait que l'humanité va vers un déclin inexorable : «L'espèce humaine dégénère : aux puissantes races des siècles passés a succédé une génération petite, maigre, chétive, chauve, myope, dont le caractère est triste, l'imagination sèche, l'esprit pauvre $»^{15}$. Plus on s'approche du tournant du $\mathrm{XX}^{\mathrm{e}}$ siècle, plus on est frappé d'une réelle crise mortelle, « avec dévaluation des valeurs et certitude que plus rien n'a de sens $»^{16}$. Paul Bourget (1852-1936) n'hésite pas à diagnostiquer que l'Europe occidentale est « malade de civilisation ${ }^{17}$, impuissante à étreindre ses chimères $»^{18}$; et Max Nordau (1849-1923) de constater non sans crainte : " nous nous trouvons actuellement au plus fort d'une grave épidémie intellectuelle, d'une sorte de peste noire de dégénérescence et d'hystérie, et il est naturel que l'on se demande de toutes parts avec angoisse : 'Que vat-il arriver ?' ${ }^{19}$.

Provins se nourrit des écrits qui font autorité dans les milieux médicaux sur ce sujet préoccupant tout en les complétant par ses propres observations critiques. Si le dramaturge est ahuri par les constats de la déchéance de la « race », il est convaincu que l'« abâtardissement » de celle-ci n'est pas exclusivement la cause de tares organiques héréditaires. Si c'était le cas, comme le démontre, entre autres, Cesare Lombroso (1835-1909), un « dégénéré » ne serait point responsable de ses défauts

${ }^{12}$ A. Liégeois, «La théologie et l'éthique sous-jacente de la psychiatrie de B.-A. Morel », Ephemerides Thelogicae Lovanienses, décembre, 1989, p. 338.

${ }^{13}$ Sans prétendre donner une liste exhaustive de tous les médecins ou écrivains, français ou étrangers, qui se sont exprimés sur le sujet de la « dégénérescence », on se limite à évoquer les noms de ceux qui ont directement inspiré le dramaturge.

${ }^{14} \mathrm{~B}$. A. Morel, Traité des dégénérescences physiques, intellectuelles et morales de l'espèce humaine, Paris, Jean-Baptiste Baillière, 1857.

${ }^{15} \mathrm{H}$. Verdé-Delisle, De la dégénérescence physique et morale de l'espèce humaine déterminée par le vaccin, Paris, Charpentier, Libraire-Éditeur, 1855, p. 5.

16 J. Russ, Le Tragique créateur. Qui a peur du nihilisme?, Paris, Armand Colin, 1998, p. 125.

${ }^{17} C f$. « il n'est pas douteux que le système nerveux de nos descendants les plus normaux aura peine à supporter l'incessante trépidation de la vie fiévreuse qui de jour en jour ébranle davantage l'humanité civilisée. Par son évolution même, le milieu social produira d'innombrables dégénérés que caractériseront surtout des stigmates psychologiques et des stigmates sociologiques », (L. Mayet, Études sur les dégénérés : les stigmates anatomiques et physiologiques de la dégénérescence et les pseudo-stigmates anatomiques et physiologiques de la criminalité, Lyon, A. Storck \& $\mathrm{C}^{\text {ie }}$, Imprimeurs-Éditeurs, 1902, p. 5).

${ }^{18}$ P. Bourget, Essais de psychologie contemporaine, Paris, Tel-Gallimard, 1993, p. 247.

${ }^{19}$ M. Nordau, Dégénérescence (L'Égotisme-Le Réalisme - Le Vingtième siècle), t. 2, trad. A. Dietrich, Paris, Félix Alcan Éditeur, 1894, p. 525. 
reconnus « innés $»^{20}$. Alors, l'écrivain ne pourrait pas décrier les travers de ceux qu'il abhorre sincèrement, mais devrait plutôt compatir au destin tragique, puisque prédéterminé et inéluctable, de ces pauvres hères atteints d'anomalies congénitales graves. Or, selon l'écrivain (de concert avec Bourget), la dégénérescence serait plutôt une « disposition d'âme $»^{21}$ de ceux qui sont moralement abaissés, ce qui n'exclut pas pour autant les difformités physiques pouvant accélérer la dégradation morale. C'est dans cette perspective que le dramaturge entame d'une manière narquoise une œuvre aux allures épiques dans laquelle il dresse un portrait déconcertant de sa génération avilie. En s'érigeant en moraliste - car tout en dépréciant la décadence, il croit avec conviction à la renaissance des valeurs, corrompues au cours du siècle - il stigmatise les fautes des puissants de ce monde qui, loin de porter le flambeau de la « santé publique », se vautrent dans la ripaille et la dépravation honteuses. Dans « les pages d'étude avant le rideau », Provins énumère les péchés capitaux des piliers de l'ordre bourgeois comme autant d'individus qui ont perdu les qualités naturelles de sa race. Premièrement, ce qui manque au " dégénéré », c'est le sens de la moralité : il se caractérise par une émotivité excessive qui ne lui permet pas de sortir de son égotisme. Puis, ayant constaté sa « débilité cérébrale », le dramaturge remarque que le "malade » a une aversion pour l'action, car privé de toute énergie, il peut s'adonner aux plaisirs n'exigeant aucun effort. Et enfin, comme celui-ci est instable psychologiquement, il perd « la notion de ce qu'est le bien et le mal, restant dominé par une préoccupation unique : celle de satisfaire ses appétits ! $\gg{ }^{22}$ Cet égoïste aux nerfs maladivement ébranlés, se moque des convenances et de la morale. Son arrogance et son mépris des autres font de lui un esclave de la sensation et, comme il rejette tout point de repère, il sombre dans l'ennui, le dégoût de lui-même et le pessimisme absolu. Face à la propagation de l'« épidémie » qui fait des ravages dans toute la nation, Provins ne peut pas rester indifférent. Étant donné que la cohorte des « dégénérés » démolit tout, «ne réédifiant jamais $»^{23}$, l'écrivain propose à travers son drame d'apporter humblement une pierre à l'édifice de la société régénérée. C'est pourquoi, l'auteur s'en prend avant tout aux politiciens cupides et aux financiers spéculateurs dont les actions appauvrissent encore plus les couches les plus démunies au profit de leurs gains personnels. Il accuse aussi les littéraires et artistes, se complaisant dans des « brouillards vagues », qui contribuent pernicieusement à la dépression et à la désagrégation de la population.

La première de la pièce, qui a eu lieu le 12 mai 1897 à la Bodinière, une ancienne scène parisienne, n'est pas passée inaperçue. Même si les représentations n'ont pas fait long feu, le drame de Provins a provoqué de vives réactions. La majorité

\footnotetext{
${ }^{20}$ C. Lombroso, L'Uomo delinquente, Milano, Hoepli, 1876.

${ }^{21}$ P. Bourget, op. cit., p. 409.

${ }^{22}$ M. Provins, Dégénérés !, Paris, G. Havard Fils Éditeur, 1897, p. 11.

${ }^{23}$ Ibid., p. 12.
} 
écrasante des critiques accueillent ce texte avec enthousiasme surtout en ce qui concerne le diagnostic virulent que l'auteur porte sur la société française. Pourtant, on lui fait quelque grief de ne pas avoir formulé explicitement de solutions pour améliorer cet état des choses. Francisque Sarcey apprécie ce "drame curieux » qui se moque ouvertement de la frivolité prétentieuse des " générations de dernier bateau ». Il le salue comme une œuvre apte à réveiller les consciences des Français : « la saynète de M. Michel Provins, ce n'est pas encore les Précieuses ridicules de Molière. N'importe ! C'est le premier coup de tocsin $»^{24}$, et le critique de constater dans un autre journal que « c'est une œuvre intéressante et digne d'être vue » qui, pourtant, ne pourrait pas être jugée à sa juste valeur par les foules à cause de sa langue venimeuse ${ }^{25}$. François Veuillot applaudit la façon avec laquelle le dramaturge présente une collection de "franches canailles », tout en la considérant comme « brutale et hardie $»^{26}$. Mais, selon le critique, tous les moyens semblent permis pour pallier la dégénérescence qui ronge la nation. Néanmoins, dans Revue du monde catholique, Veuillot déplore que l'auteur n'ait pas formellement démontré les causes de cette dégénérescence et n'ait pas proposé de remèdes concrets, tout en constatant que « ce mal ne guérira que par la foi retrouvée $»^{27}$. À propos du texte de Provins, Hyppolyte Lemaire parle d' " un cliquetis continuel de mots brillants et d'idées hardies $\gg^{28}$ qui non seulement amuse le public, mais avant tout incite à la réflexion, tandis qu'Émile Faguet caractérise la pièce comme une « comédie cruelle dans toute son ampleur [et] dans tout son épanouissement $»^{29}$ qui est capable d'ébranler la sérénité des spectateurs. Malgré le cynisme de l'auteur, Edmond Stoullig loue « l'esprit, le brio, la vigueur et l'audace de son œuvre $»^{30}$ qui ne laisse pas indifférent. La pièce, qui a été reprise le 6 mai 1899, au Théâtre du Gymnase, a aussi bouleversé le public. En témoigne Paul-Émile Chevalier selon lequel la représentation a laissé « une lourde impression d'outrance et de mauvais écœurement $\|^{31}$, tandis que Jean Thorel reproche à l'auteur d'être un simple satiriste qui décrit ses personnages comme des «mufles » plutôt que comme des dégénérés : « ce sont des brutes, presque primitives, qui agissent avec violence et une 'énergie' sauvages. Ils sont le contraire de dégénérés $\star^{32}$. En dépit de cette observation, la pièce a remporté un succès considérable en Italie où Dégénérés ! a été accueilli,

\footnotetext{
${ }^{24}$ F. Sarcey, « Précieux \& décadents », Le XIX siècle, 16 juin 1897, p. 1.

${ }^{25}$ F. Sarcey, « Chronique théâtrale », Le Temps, 17 mai 1897, p. 2.

${ }^{26}$ F. Veuillot, « Dégénérés », L’Univers, 18 août 1897, p. 1.

${ }^{27}$ F. Veuillot, «Le théâtre et les idées. Dégénérés par Michel Provins », Revue du monde catholique, t. 132,1897 , p. 118 .

${ }^{28}$ H. Lemaire, "Théâtres », Le Monde illustré, 22 mai 1897, p. 338.

${ }^{29}$ É. Faguet, «La semaine dramatique », Journal des débats politiques et littéraires, 17 mai 1897, p. 1.

${ }^{30}$ E. Stoullig, « Chronique dramatique », Le Monde artiste, n 20, 16 mai 1897, p. 310.

${ }^{31}$ P.- É. Chevalier, « Semaine théâtrale », Le Ménestrel, nº 20, 14 mai 1899, p. 155.

32 J. Thorel, «Chronique dramatique », Le XIXe siècle, 8 mai 1899, p. 2.
} 
aux dires d'Alfred Delilia, avec une « ovation enthousiaste faite par le public à l'auteur et aux excellents artistes $\gg{ }^{33}$.

Provins semble nous introduire dans son hôpital psychiatrique où il règne en sévère clinicien. Ses patients semblent effectivement gravement malades. Dès les premières répliques, ces bourgeois apathiques et amorphes n'hésitent point à se considérer comme des minables qui se meuvent dans ce monde comme des ombres. Ils sont bien conscients de leur " dégénérescence », mais incapables de lutter contre l'inertie, ils s'enlisent avec une certaine volupté dans cet état d'infériorité. Cependant, malgré la prise de conscience de leurs défauts, ces " épaves humaines » s'accordent la permission d'exercer de hautes fonctions dans le système capitaliste. De fait, ces « décadents » sont décrits comme des pervers qui se plaisent dans leur inconduite condamnable. De prime abord, ils se déclarent sur un ton sarcastique et ironique représentants de l'humanité déchue. Voici quelques remarques à l'emporte-pièce qui témoignent de la gravité de leur état mental. Barral, psychologue aux nerfs fragiles, constate tristement : " nous sommes des dégénérés, des déséquilibrés, des neurasthéniques! $\gg^{34}$ tandis que Livrary, homme d'affaires qui s'est enrichi par la spéculation, s'écrie plein d'amertume : «Ah ! Nous sommes bien de la décadence et, qui pis est, de la décadence analytique et raisonnée ! $\aleph^{35}$, ce qui sera résumé par les propos d'un Chambard, débutant en politique : "Non plus, hélas! de cette superbe décadence latine ${ }^{36}$ qui avait au moins le courage de l'orgie $»^{37}$. Tous les compagnons de débauche ressassent leur condition qu'ils auraient, selon eux, héritée de leurs parents, comme s'ils voulaient rejeter sur leurs procréateurs la responsabilité de leur avilissement. Dans ce contexte, ils peuvent s'adonner à toutes sortes d'extravagances et de bassesses afin d'amoindrir les effets secondaires de l'ennui qui les ronge douloureusement. Dégradés par l'abus d'alcool et d'autres drogues, ils sont condamnés à se régaler de tisanes et de cuisine simple et légère. Ils fantasment encore sur leurs potentiels exploits (entre autres, l'extermination des Chinois, la recherche de plaisirs sensuels abrutissants) tout en restant inaptes à imaginer quelque chose de constructif. Ils ressemblent, toute proportion gardée, aux fantoches de Jarry auxquels on a enlevé le " caractère " pour le remplacer par une " outrance », un masque, incarnation de leurs vices : la cupidité, la goinfrerie et la lâcheté. Privés de traits psychologiques, les déchus rappellent tout autant les nihilistes indifférents à tout discernement moral, idée que le dramaturge semble partager avec Bourget. Les deux écrivains expriment à travers leurs personnages l'état d'esprit « immoraliste » qui symbolise

\footnotetext{
${ }^{33}$ A. Delilia, « Courrier des Théâtres », Le Figaro, 23 novembre 1899, p. 4.

${ }^{34}$ M. Provins, Dégénérés !, op. cit., p. 42.

${ }^{35}$ Ibid., p. 41-42.

${ }^{36}$ Cf. M.-F. David-de Palacio, Reviviscences romaines. La latinité au miroir de l'esprit fin-de-siècle, Berne, Peter Lang, 2005.

${ }^{37}$ M. Provins, Dégénérés !, op. cit, p. 41.
} 
l'anéantissement des valeurs. Et pour cause, car pour le nihiliste « le bien et le mal, la beauté et la laideur, le vice et la vertu lui paraissent des objets de simple curiosité [...] Pour lui, rien n'est vrai, rien n'est faux, rien n'est moral, rien n'est immoral. C'est un égoïste subtil et raffiné $»^{38}$.

Néanmoins, la seule chose qui puisse encore inciter ces vauriens à une quelconque action, c'est la volonté de s'enrichir. Ce n'est qu'à cela qu'ils tiennent farouchement. Comme la carrière politique peut assurer une gratification importante tout en permettant d'assouvir ses instincts parfois morbides, Chambard désire trouver un travail au Palais Bourbon. Au demeurant, il est persuadé que la tâche n'est point difficile tout en soulignant qu'il connaît la rhétorique des parlementaires. De plus, il dédaigne les préoccupations d'ordre moral, ce qui l'amène à prétendre avec un cynisme aussi grotesque qu'effrayant :

Croyez-vous à la correction de tous ceux qui, ne pouvant pas donner du pain au peuple, lui promettent des confitures ? Non, n'est-ce pas... Eh bien, il faut faire comme eux, s'emparer du pouvoir par tous les moyens possibles... C'est, pour les classes dirigeantes, un devoir de défense sociale ! ${ }^{39}$

Dès lors, le candidat désireux d'exercer les fonctions d'un député « respectable» doit tenir devant ses électeurs des propos résolument ineptes ${ }^{40}$. Voici un exemple de parodie de la logomachie politique :

Chambard : J'estime tout d'abord qu'il faudra réaliser des économies compatibles avec le bon fonctionnement des services publics... je préconiserai les mesures qui seraient susceptibles de frapper la richesse acquise...

Barral (à mi-voix) : Radical!

Chambard : (continuant) : Tout en respectant les droits de la propriété, qui n'est autre chose que du travail accumulé !...

Barral (de même) : Conservateur!

${ }^{38}$ P. Bourget, Le Disciple, Paris, Librairie Plon, 1901, p. 12.

${ }^{39}$ M. Provins, op. cit., p. 50.

40 «L'électeur tient à ce qu'on flatte ses convoitises et ses vanités; il faut l'accabler des plus extravagantes flagorneries, ne pas hésiter à lui faire les plus fantastiques promesses. S'il est ouvrier, on ne saurait trop injurier et flétrir ses patrons. Quant au candidat adverse, on doit tâcher de l'écraser en établissant par affirmation, répétition et contagion qu'il est le dernier des gredins, et que personne n'ignore qu'il a commis plusieurs crimes. Inutile, bien entendu, de chercher aucun semblant de preuve. Si 'adversaire connaît mal la psychologie des foules, il essaiera de se justifier par des arguments, au lieu de se borner à répondre aux affirmations par d'autres affirmations; et il n'aura dès lors aucune chance de triompher. - Le programme écrit du candidat ne doit pas être trop catégorique, parce que ses adversaires pourraient le lui opposer plus tard ; mais son programme verbal ne saurait être trop excessif. Les réformes les plus considérables peuvent être promises sans crainte. Sur le moment, ces exagérations produisent beaucoup d'effet, et pour l'avenir elles n'engagent en rien. Il est d'observation constante, en effet, que l'électeur ne s'est jamais préoccupé de savoir jusqu'à quel point l'élu a suivi la profession de foi acclamée, et sur laquelle l'élection est supposée avoir eu lieu » (G. Le Bon, Psychologie des foules, Paris, Félix Alcan Éditeur, 1895, p. 108). 
Chambard (continuant) : En matière religieuse je réclamerai énergiquement la prépondérance de la société civile...

Barral (de même) : Anticlérical !

Chambard (continuant) Restant néanmoins animé d'un sincère esprit de tolérance pour les idées religieuses, si respectables, d'un grand nombre de nos concitoyens...

Barral (de même) : Réactionnaire !

Chambard (continuant) : En matière économique, je veux que les travailleurs aient droit, dans tous les bénéfices de l'exploitation, à une participation largement rémunératrice...

Barral (de même) : Socialiste ! ...

Chambard (continuant) : En somme, je soutiendrai une politique à la fois progressiste, sagement libérale, et résolument réformatrice ? ${ }^{41}$

Il en est de même dans le dialogue Genèse d'un socialiste, où un professeur rêve depuis longtemps d'un poste dans un lycée de première classe. Ayant rendu des services électoraux à un député, il s'attend enfin à la récompense promise, mais il devra éprouver une nouvelle désillusion. Dès lors, meurtri par la vilenie du politicard, il sombre dans la tristesse. Le cabaretier Jumac incite son collègue à la vengeance : en devenant socialiste, celui-ci ne punirait pas seulement son offenseur mais, de plus, il se garantirait une belle vie bien rémunérée. L'instituteur possède le talent de la parole mais il lui manque " la psychologie populaire ", c'est pourquoi, l'habitué des estaminets se charge de l'éducation du potentiel parlementaire. Jumac explique ${ }^{42}$ au futur candidat révolutionnaire les méandres de la vie politique (« dans la vie on n'a le choix qu'entre deux solutions : manger les autres, ou être mangé par eux $\rangle^{43}$ ), tout en le renseignant sur l'art rhétorique et sur la façon de se comporter face aux masses. Pour gagner les élections, il ne faut point penser aux biens des hommes, mais à leurs votes : un homme de foi doit promettre au peuple plus que ses rivaux politiques :

Laisse ton esprit broder les plus fantastiques improvisations : on te croira toujours ! Dis qu'on prendra, pour leur jeter en pâture, l'or et les palais des capitalistes, que les terres et les mines seront à eux, que les riches asservis deviendront leurs domestiques, que les fontaines d'eau se changeront en ruisseaux de vin, que, sous l'œil bienveillant de l'ÉtatProvidence, toute la masse ouvrière pourra se gorger des jouissances humaines ! Surexcite ton imagination, brode, invente, déclame et ne recule devant aucune absurdité : en face de l'homme qui évoque le rêve du bonheur, la bêtise du peuple est insondable !4

Une fois au pouvoir, Morsec pourra continuer sa comédie ne pensant qu'aux biens matériels. Provins déplore les vrais motifs qui poussent les hommes à la carrière politique. Il les considère comme dangereux car ils ont oublié les idéaux qui devraient

\footnotetext{
${ }^{41}$ M. Provins, op. cit., p. 69-70.

${ }^{42}$ Cette scène annonce l'épisode de La Résistible Ascension d'Arturo Ui (1941) de Brecht, au cours duquel un vieux comédien shakespearien enseigne au gangster à parler devant la foule.

${ }^{43}$ M. Provins, op. cit., p. 174-175.

${ }^{44}$ Ibid., p. 175-176.
} 
accompagner les élus dans leurs actes pour le bien commun. De fait, les représentants de la société remplacent la foi en l'homme et en Dieu par une nouvelle religion : celle de l'Argent. Dans un éloge paradoxal digne du meilleur Mirbeau, Provins dénonce l'égoïsme et la stupidité des parvenus, ce qui le rapproche des auteurs tels que Brieux (Les Bienfaiteurs) ou Bernard Shaw (La Commandante Barbara ${ }^{45}$ :

\begin{abstract}
Voilà pourquoi nous devons vénérer notre maître, l'argent : c'est qu'aujourd'hui, dans ce siècle niveleur, au milieu de cette foule de médiocres et d'impuissants, il représente la seule royauté qui demeure ! Grâce à lui, toutes les puissances du monde sont à nos pieds : le talent, l'intelligence, les honneurs, la gloire, nous pouvons tout acheter. Il nous permet, mesdames, d'ajouter à vos sourires des perles, des diamants, et de vous rendre superbement le culte qui vous est dû. Enfin, il ne faut rien oublier - pas même les malheureux - et je veux saluer le beau privilège de la richesse : la charité ! ${ }^{46}$
\end{abstract}

Si les politiciens, brasseurs d'affaires et banquiers vénaux sont la cible préférée de Provins, il n'oublie pas pour autant le rôle dévastateur des littéraires et des artistes sur la santé morale de la société. Une fois de plus, l'écrivain s'inspire des hérauts de la médecine contemporaine en la matière. À ce propos il serait légitime de citer Nordau qui, dans un ouvrage dédicacé à Lombroso, déclare :

les dégénérés ne sont pas toujours des criminels, des prostituées, des anarchistes ou des fous déclarés ; ils sont maintes fois des écrivains et des artistes. Mais ces derniers présentent les mêmes traits intellectuels - et le plus souvent somatiques - que les membres de la même famille anthropologique qui satisfont leurs instincts malsains avec le surin de l'assassin ou la cartouche du dynamiteur, au lieu de les satisfaire avec la plume et le pinceau ${ }^{47}$.

En effet, dans le dernier dialogue Un salon parisien en 1897 (avec le soustitre « pour servir à l'histoire de mon temps »), le dramaturge s'en prend aux milieux intellectuels, constitués de préférence par des snobs et des artistes manqués qui, rongés par les nouveautés venant de l'étranger, vénèrent le culte de l'obscur, le contraire de l'âme française ${ }^{48}$. L'auteur méprise ouvertement " l'école du flou » au rang de laquelle il met pêle-mêle les symbolistes, les mystiques, les naturalistes ou les décadents. Il exècre « le Tolstoïsme », «l'Ibsenisme », déprécie les œuvres de D'Annunzio et de Sudermann, en revanche, il réserve les lettres de noblesse à Lamartine, Victor Hugo et Balzac. En peinture il n'estime guère les impressionnistes, les tachistes, les pointillistes tandis qu'en musique Wagner est en butte à ses sarcasmes. Pourtant, les adeptes d'un nouvel art s'extasient devant

${ }^{45} C f$. Guy Ducrey, «Le Théâtre contre la charité. Octave Mirbeau, Eugène Brieux, Bernard Shaw », Littératures 64, 2011, p. 167-184.

${ }^{46}$ M. Provins, op. cit., p. 164.

${ }^{47}$ M. Nordau, Dégénérescence (Fin de siècle - le Mysticisme), t. 1, trad. A. Dietrich, Paris, Félix Alcan Éditeur, 1894, p. 3-4.

${ }^{48}$ Selon Provins, les Français restaient presque ensorcelés particulièrement par tout ce qui était allemand ou anglais. Ils n'étaient pas moins admiratifs à l'égard de l'« esprit slave ». 
tout ce qui paraît abscons, ce dont témoignent si bien leurs propos boursouflés, difficiles à comprendre, aux dires de l'écrivain, par les hommes sains d'esprit. Ainsi, Provins n'hésite pas à caricaturer le langage ampoulé et extravagant des prosélytes de certains « cercles décadents », tout en attaquant ouvertement Mallarmé :

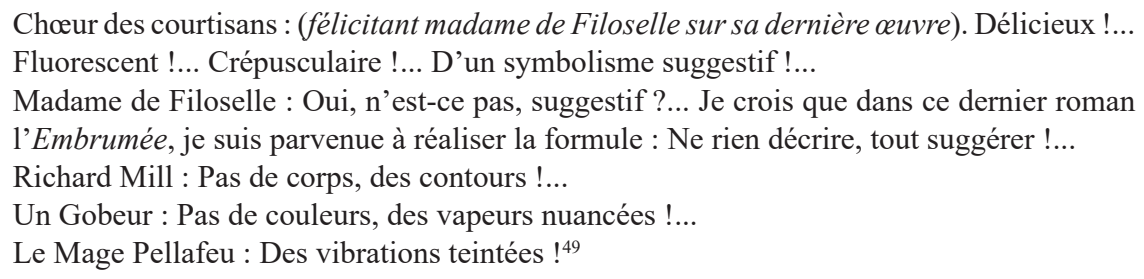

L'auteur introduit dans la pièce le personnage du Naïf qui, n'ayant pas encore succombé aux charmes douteux de l'art fin de siècle, perçoit les invités comme des marionnettes aussi déraisonnables qu'abjectes, mais c'est à l'Initié de commenter malicieusement l'état précaire de l'intelligence de cette " élite dégénérée ». C'est lui qui, en qualité de porte-parole de l'auteur, dénonce la "gangrène intellectuelle » des habitués de salons où l'on rencontre « combinés à différentes doses, le noble, le républicain, le rallié, le socialiste, le financier, l'industriel [...] le wagnérien, le décadent, le symboliste, le coureur de dots et la demi-vierge ${ }^{50}$, la divorcée et la déraillée $»^{51}$. Celui qui mérite le blâme, c'est avant tout l'étranger, ce sauvage qui empoisonne l'esprit français par les « brouillards de ses littératures » afin d'assouvir ses obsessions matérielles :

Étant chez nous, il a vidé nos poches pour organiser, chez lui, la concurrence à tous nos produits. Installé et engraissé, le Barbare a envahi le domaine intellectuel : les théâtres, la musique, les livres, la philosophie ; on ne pense, on n'entend, on ne jure que par lui. Ce qu'il ne subjugue pas, il l'emporte... comme les œuvres de nos artistes, les noms de notre noblesse, les voix de nos actrices, les... caresses de nos jolies femmes ${ }^{52}$.

L'émotion première, qui imprègne toute la pièce, est le mépris du dramaturge au sujet des vicieux mondains. Ceux-ci ne cherchent que des sensations égoïstes et nihilistes, car au fond ils sont « des brutes toutes primitives [et] des singes $»^{53}$ qui infectent gravement de leur dégénérescence les nouvelles générations. Alors, Provins a recours aux mots forts tout en dénigrant les responsables de cet état

${ }^{49}$ M. Provins, op. cit., p. 230.

${ }^{50}$ Allusion évidente aux Demi-Vierges de Marcel Prévost, roman publié par Alphonse Lemerre en 1894.

${ }^{51}$ Ibid., p. 248.

${ }^{52}$ Ibid., p. 248.

${ }^{53}$ J. Lemaître, Impressions de théâtre, Société française d'imprimerie et de librairie, " Nouvelle Bibliothèque littéraire », Paris, 1898, http://obvil.sorbonne-universite.site/corpus/critique/lemaitre impressions/10/ : consulté le 9.09.2018. 
de choses. Il sait bien que les éléments farcesques de son œuvre peuvent toucher d'une manière plus efficace l'intelligence critique du public.

Accabler les bourgeois d'injures serait-il un acte d'émancipation des classes opprimées ou juste un appel à la régénération de la classe dirigeante ? Ce serait au lecteur / spectateur de choisir l'interprétation qui lui convient le mieux. Quoi qu'il en soit, Provins est un « réactionnaire éclairé » qui " présente [dans sa pièce] des 'âmes vermoulues' et abouliques, celles-là même que stigmatisait, en 1894, le fameux Dégénérescence de Max Nordau $»^{54}$.

Les propos critiques qui émaillent cette pièce aux accents accusateurs devaient bouleverser. Que l'on ajoute à l'outrance des énoncés, une écriture sarcastique qui n'est autre que l'expression du mépris à l'égard des magnats pernicieux. Néanmoins, malgré un ton péremptoire et une incrimination sans complaisances, Provins ne désire point souscrire aux thèses anarchistes de Jean Grave. De fait, si on faisait abstraction des commentaires de l'écrivain, on pourrait fautivement constater que Les Dégénérés ! est un drame issu de la plume d'un libertaire rigoureux, mais l'auteur de Tendresses futiles se veut résolument le défenseur des valeurs traditionnelles minées par une crise mortelle se manifestant à travers l'esprit décadent néfaste. Secouer les consciences morales pour protéger l'ordre social et la doxa conservatrice contre la propagation de la dégénérescence létale : voici son mot d'ordre combatif. C'est pour cette raison qu'il doit être farouche, recourir aux « énormités burlesques 》 et acerbes afin de faire sortir le citoyen de son ankylose décadente. Selon lui, la vraie mission du théâtre n'est pas de divertir, donc de procurer une joie soporifique ou un plaisir violant les principes de la morale ${ }^{55}$, mais au contraire, de perturber, car il doit « aborder l'étude des névroses, fouiller les plaies sociales. Tant pis si quelque pestilence s'en dégage : c'est à force de montrer la hideur du mal que s'imposera la nécessité de l'antisepsie $»^{56}$. Provins désire obéir à l'impératif

${ }^{54}$ R. Raymond, préface à C. Mauclair, Le Soleil des morts, Paris / Genève, Slatkine Reprints, 1979, p. IV.

${ }^{55} \mathrm{Cf}$. « Comment nier que la dissolution des mœurs s'alimente dans nos cités, au milieu des théâtres où se jouent de prétendues comédies, vaudevilles, comédies-vaudevilles, etc., et tout ce fatras de pièces bâtardes, aussi intolérables par la faiblesse de conception que dégoûtantes par leur immoralité. C'est là que le jeune homme va recevoir les premières leçons de débauche, et s'habituer à des vices qui corrompent son cœur et ruinent sa santé. La licence, pour ne pas dire le cynisme du langage et des gestes, agacent sa chair, stimulent ses appétits et produisent dans tout son être une exaltation passionnée à laquelle il faut obéir. Poursuivi la nuit et le jour par des images séduisantes et des désirs que sollicitent déjà son âge et sa nature, il s'abandonne à des plaisirs solitaires qui minent son corps et tuent son intelligence " (H. Bonnaîre, Influence du théâtre sur la santé publique, Imprimerie de Didot le Jeune, 1834, p. 12-13).

${ }^{56}$ M. Provins, op. cit., p. 22. 
de conjurer le danger public d'un mal qui ronge la société de son époque. Ayant déterminé la « dégénérescence » d'après les symptômes et l'examen quasi clinique de ses contemporains, le dramaturge croit fermement à la renaissance de l'humanité et se permet de proposer une panacée qui se résume en trois mots : la volonté, le devoir, l'idéal. Il faut revenir aux origines « divines » de l'homme, réveiller en lui l'idée de la responsabilité vis-à-vis d'autrui, chérir les valeurs religieuses solides ${ }^{57}$ pour pouvoir remédier à la dégradation morale. Ainsi exhorte-t-il ses compatriotes à s'en repentir :

Il faut que les hommes sains, que la grande masse des braves gens, toujours trop passifs, que tous ceux qui, par la parole, par la plume, par l'exemple, par une supériorité quelconque, disposent d'une influence, se réunissent pour purger le monde des dégénérés nuisibles, pour étouffer leurs doctrines, pour détruire les germes contagieux qui émanent de leurs actes ! Il faut une révolte de l'espèce, pour que les anti-sociaux disparaissent $!^{58}$

\section{Bibliographie}

Autrand, Michel, Le Théâtre en France de 1870 à 1914, Paris, Honoré Champion, 2006

Beaudu, Édouard, « Nouvelle Fantaisies », La Chronique des livres, 10-15 juillet 1904

Bonnaîre, Hubert, Influence du théâtre sur la santé publique, Paris, Imprimerie de Didot le Jeune, 1834 Bourget, Paul, Le Disciple, Paris, Librairie Plon, 1901

Bourget, Paul, Essais de psychologie contemporaine, Paris, Tel-Gallimard, 1993

Chevalier, Paul-Émile, « Semaine théâtrale », Le Ménestrel, n 20, 14 mai 1899

David-de Palacio, Marie-France, Reviviscences romaines. La latinité au miroir de l'esprit fin-de-siècle, Berne, Peter Lang, 2005

Delilia, Alfred, « Courrier des Théâtres », Le Figaro, 23 novembre 1899

Ducrey, Guy, " Le Théâtre contre la charité. Octave Mirbeau, Eugène Brieux, Bernard Shaw », Littératures 64, 2011, p. 167-184

Ebstein, Jonny et al., Le Théâtre de contestation sociale autour de 1900, Paris, PUBLISUD, 1991

Ebstein, Jonny et al., Au temps de l'anarchie, un théâtre de combat : 1880-1914, Paris, Séguier / Archimbaud, 2001

Faguet, Émile, « La semaine dramatique », Journal des débats politiques et littéraires, 17 mai 1897

Le Bon, Gustave, Psychologie des foules, Paris, Félix Alcan Éditeur, 1895

Lemaire, Hyppolyte, « Théâtres », Le Monde illustré, 22 mai 1897

Lemaître, Jules, Impressions de théâtre, Société française d'imprimerie et de librairie, " Nouvelle Bibliothèque littéraire ", Paris, 1898, http://obvil.sorbonne-universite.site/corpus/critique/ lemaitre_impressions/10/; consulté le 9.09.2018

Liégeois, Axel, « La théologie et l'éthique sous-jacente de la psychiatrie de B.-A. Morel », Ephemerides Thelogicae Lovanienses, décembre, 1989, p. 330-357.

Lombroso, Cesare, L'Uomo delinquente, Milano, Hoepli, 1876

Mauclair, Camille, Le Soleil des morts, Genève, Slatkine Reprints, 1979

${ }^{57}$ Provins semble peu convaincu en ce qui concerne la véracité de l'enseignement catholique, mais, face à la dégénérescence, il va faire feu de tout bois afin de sauvegarder l'ordre et la santé publics.

${ }^{58}$ M. Provins, op. cit., p. 32. 
Mayet, Lucien, Études sur les dégénérés : les stigmates anatomiques et physiologiques de la dégénérescence et les pseudo-stigmates anatomiques et physiologiques de la criminalité, Lyon, A. Storck \& $\mathrm{C}^{\mathrm{ie}}$, Imprimeurs-Éditeurs, 1902

Morel, Bénédict Augustin, Traité des dégénérescences physiques, intellectuelles et morales de l'espèce humaine, Paris, Jean-Baptiste Baillière, 1857

Morel, Bénédict Augustin, Traité des maladies mentales, Paris, Librairie Victor Masson, 1860

Nordau, Max, Dégénérescence (Fin de siècle - le Mysticisme), t. 1, trad. A. Dietrich, Paris, Félix Alcan Éditeur, 1894

Nordau, Max, Dégénérescence (L'Égotisme - Le Réalisme - Le Vingtième siècle), t. 2, trad. A. Dietrich, Paris, Félix Alcan Éditeur, 1894

Provins, Michel, Dégénérés !, Paris, G. Havard Fils Éditeur, 1897

Provins, Michel, La Femme d'aujourd'hui, Paris, Victor-Havard, 1895

Provins, Michel, «L'école des flirts », L'Art et la scène, nº 12, 1897

Russ, Jacqueline, Le Tragique créateur. Qui a peur du nihilisme?, Paris, Armand Colin, 1998

Sarcey, Francisque, «Précieux \& décadents », Le XIX` siècle, 16 juin 1897

Sarcey, Francisque, « Chronique théâtrale », Le Temps, 17 mai 1897

Stoullig, Edmond, « Chronique dramatique », Le Monde artiste, n 20, 16 mai 1897

Thorel, Jean, "Chronique dramatique », Le XIX` siècle, 8 mai 1899

Verdé-Delisle, Henri, De la dégénérescence physique et morale de l'espèce humaine déterminée par le vaccin, Paris, Charpentier, Libraire-Éditeur, 1855

Veuillot, François, « Dégénérés », L’Univers, 18 août 1897

Veuillot, François, "Le théâtre et les idées. Dégénérés par Michel Provins », Revue du monde catholique, t. 132,1897

Tomasz Kaczmarek enseigne la langue et la littérature (italienne et française) à l'Université de Łódź. Thèse sur l'œuvre de Henri-René Lenormand. Habilitation sur le personnage dans le théâtre français face à la tradition de l'expressionnisme européen. Publications sur le théâtre français, italien et polonais dans le contexte des avant-gardes du $\mathrm{XX}^{\mathrm{e}}$ siècle.

\begin{tabular}{|l|l|}
\hline cc Creative & $\begin{array}{l}\text { Co by the author, licensee Łódź University - Łódź University Press, } \\
\text { Łódź, Poland. This article is an open access article distributed under } \\
\text { the terms and conditions of the Creative Commons Attribution license } \\
\text { CC-BY-NC-ND 4.0 (https://creativecommons.org/licenses/by-nc-nd/4.0/) }\end{array}$ \\
\cline { 2 - 2 } & Received: 2019-01-15; Accepted: 2021-03-29 \\
\hline
\end{tabular}

